\title{
VMS Mean and FFD VM Allocation Algorithm for Cloud Datacenter
}

\author{
Sreenivasa B.L, S Sathyanarayana
}

\begin{abstract}
The datacenter power consumption is increasing speedily, because of the scalable and dynamic provisioning of resources to the remote consumers. Not efficiently utilizing the resource of host machine is the main reason for increase in power consumption. By efficiently placing the number of virtual machine in fewer active host and shutdown the inactive host could reduce the consumption of power in datacenter. In this paper VMs Mean and FFD Bin Packing Virtual Machine Placement Algorithm is proposed.FFD preprocess VM's and Host's by sorting all the VM's by MIPS and Host's by their power. To place the VM's in host machine VMs Mean method is used which finds the lowest CPU utilizing VM. The main objective is to increase the host machine utilization by efficiently placing the VM's into few active Host's and also reduced the power consumption. To test this algorithm CloudSim Toolkit is been used. The parameter such as EC, SLAV, PDM, ESV, VM migration and SLATAH is used as a metric to evaluate the Algorithm efficiency.
\end{abstract}

Keywords : Energy Consumption (EC), SLA Violation (SLAV), performance degradation due to migration (PDM), Energy SLA Violation (ESV), VM migration and SLA Violation Time per active host (SLATAH), First Fit Decreasing (FFD), Virtual machine (VM).

\section{INTRODUCTION}

$\mathrm{W}$ ith the internet facility the Cloud consumer are able to manage and access the data without constructing any infrastructure resource's at consumer locality. Consumer can access their resources from any locality and need not worry about maintenance and security issues. The main features of cloud computing is sharing of cloud resource between multiple consumers by using the technique of Virtualizations. Virtualization maximizes the host machine resource utilization. Virtualization increases in security,virtual machine of each have individual security policy separated by firewall rules instead on host machine. Virtual machine importing and migration is easy. In virtualization, every consumer's, one virtual machine is assigned which executes on host machine present in datacenter. In-efficient utilization of host machine resource leads to wastage of power consumed by host machine. The placement of VM's to the suitable host machine is a critical process.

Revised Manuscript Received on January 30, 2020.

* Correspondence Author

Sreenivasa B.L, Research Scholar bharathiar university Coimbatore and also works as an Asst professor in AIMIT, St Aloysius College, Mangalore.. Email: srinivasbl.vas@ gmail.com

Dr. S Sathyanarayana, First Grade Womens College Mysore, Karnataka India. Email: ssn_mys@yahoo.com
The Virtual Machine placement (VMP) algorithm assigns/consolidate VM's to Host machines. Efficiently assigning of maximum virtual machine to fewer number of host machine leads to reduced power consumption. This improves the maximum utilization of host machine. The idle/inactive host machine are shutdown to low power mode.

In this paper VMs Mean and FFD Bin Packing virtual machine placement algorithms is proposed. FFD preprocess VM's and Host's by sorting all the VM's by MIPS and Host's by their power. To place the VM's in host machine VMs Mean method is used which finds the lowest CPU utilizing VM. The main objective is to increase the host machine utilization by efficiently placing the VM's into few active Host's and also reduced the power consumption.

The remaining paper is planned as: Section II discuss the related work to this research. Section III discussion on working of VMs Mean and FFD VMP algorithm. Section IV explains the simulation setup, metrics for measuring the performance, results and analysis. Section V concludes this research.

\section{RELATED WORK}

Cloud datacenter are growing in massive scale and complications are also increasing parallel to satisfy the consumer demands. In daywise operations cloud datacenter consume enormous amount of power, it also includes the infrastructure resource cooling. Decrease in Energy consumption, maximizing the resource utilization and providing quality of services to the cloud consumers. In this paper the main work is on Virtual Machine Placement, and few works related to it is reviewed in this section.

Garima Batra et al [1] proposed Best Fit Sharing and Power Aware VM placement algorithm. The algorithm focus on two factors one is placement of VM on a corresponding host machine - increase in power consumption and second is memory sharing or say page sharing are taken into consideration. The algorithm works better than indigenous power aware and sharing aware algorithms for the randomly generated data set.

Zhou et al [2] proposed Adaptive three-threshold energy-aware algorithm (ATEA) Virtual Machine placement algorithm main goal is reducing energy consumption and SLA violation. The datacenter host are divided into four categories: host with tiny load, hosts with medium load, hosts with modest load, and hosts with dense load. 
ATEA migrates VMs on dense loaded or tiny-loaded hoststo lightly loaded hosts, while the VMs on medium loaded and modest loaded hosts remain unchanged. The algorithms prove that dynamic thresholds are more power efficient than fixed threshold.

Jing et al [3] proposed a heuristics-based VM allocation algorithm.VMs running on the similar host machine, based on the CPU utilization, if they have correlations high is likely to generate overloading events and SLA violations even though the host machine has not reached the critical stage. To avoid such violations of SLA and to preservea reduced energy consumption. In the heuristics-based Virtual machine allocation algorithm, incorporate both the utilization levels of hosts and resourceutilization correlations among co-located VMs. Using this proposed algorithm, a lower heuristic value indicates a moredesirable operating environment for both VMs and hosts.

Aneeba Khalil et al [4] proposed two heuristic based Vector Bin Packing algorithms called FFDmean and FFDmedian Virtual MachinePlacement. Firsts itsorts all the VMs in the order of descending considering VMs size. It's difficult to calculate the VMs size because of multidimensional properties of VMs. For this reason, FFDmean and FFDmedian usedto estimate the VM size. The algorithms main aim is to consume the host machines resources efficiently so that the required number ofhost machines for accommodating of all VMs can be reduced.

Pradeep et al [5] proposed power and data aware best fit algorithm for selecting next Virtual Machine with energy and data constraints. The improvised algorithm emphasizes more on energy consumption and data in datacenters. BFD algorithm takes both power and data constraint as input,to better the energyconsumption and utilization of resource. First the VMs are arranged in the order of decreasing for their CPU utilization and each VM is allotted to a host machine that reduces the consumption of energy by proper allocating resources and data awareness.

Anton beloglazov et al [6] proposed power-aware assignment heuristics provision data center resources to client applications ina way that improves power consumption efficiency of the data center, while providing the negotiated Quality ofService (QoS).The Modified Best Fit Decreasing(MBFD) algorithms, VMs all are arranged in order of decreasing for their present CPU utilizations, and assigns each Virtual Machine to a host machine thatdelivers the minimumriseinpower consumption. This permits leveraging the heterogeneity of resourcesby selecting the most power-efficient nodes first. This methodleads to a considerable reduction of power consumption in Clouddata centers when compared to resource allocation using static techniques.

Lei shi et al [7] proposed an integer linear programming model for the optimal VM placement with the objectives of minimizing the active PMs and number of migrations for PM consolidations. The author Lei shi et al [7] designed and evaluated a number of bin packing algorithms using different sorting strategies to determine firstly which one is most effective at consolidating VMs in data center enabling unused PMs to be shutdown. Furthermore, various strategies were developed to pack dynamic VM requests in the most efficient way. Each of the various bin packing algorithms offered some improvement in the number of PMs which could be powered off due to consolidation, even when the number of VM migrations needed for this consolidation was also taken into account. PercentageUtil and AbsoluteCapacity are the two algorithm which performed well. In both cases, the host machinesbased on the given metric (utilization and capacity, respectively) they are sorted, and then the algorithm tries to free smallest physical machine by migrating the VMs to the biggest physical machines in an iterative method.

\section{VMS MEAN AND FFD ALGORITHM}

In this subsection, a VMs Mean and FFD Algorithm is designed as a solution for the Virtual Machine placement problem. The host machine provisioned by the cloud datacenter can either be in active or shutdown state. An active host machine is considered for allocation by the algorithm. A shutdown host machine is not considered for the allocation by the algorithm but depending on the situation it can be considered.

To design an algorithm the factor considered here is the placement of a virtual machine on a suitable host machine with energy efficient CPU resource utilization.First all the Virtual machines are sorted in the order of decreasing MIPS. Mean for migrating Virtual machine List is calculated using equation (1) and each Virtual machine with lower CPU utilization is sent to host machine for the allocation. Then the host machine is sorted in the decreasing order of power consumption. The smaller energy consumption of host machine is selected for the further process. The host machine is checked for the underutilized or over-utilized, if the host machine has enough resource then the Virtual machine is allocated to it.

After sorting the migrating Virtual machine List inthe order of decreasing MIPS. Mean is calculated using equation (1). The larger the mean the larger the CPU utilization.

$$
\text { meanVMList }=\left(\sum_{k=0}^{n} V M^{k}\right) / \text { VMListSize }
$$

TABLE 1: SYMBOLIZATION TABLE

\begin{tabular}{|l|l|}
\hline EXPRESSION & DESCRIPTION \\
\hline vmsToMigrate & $\begin{array}{l}\text { LIST OF MIGRATING } \\
\text { VM'S }\end{array}$ \\
\hline VMListSize & $\begin{array}{l}\text { NUMBER OF VM'S IN } \\
\text { vmsToMigrate }\end{array}$ \\
\hline meanVMList & $\begin{array}{l}\text { Mean of VMsCPU } \\
\text { utilization in } \\
\text { vmsToMigrate }\end{array}$ \\
\hline totalVMUtilization & $\begin{array}{l}\text { Total CPU utilization of } \\
\text { VMSs }\end{array}$ \\
\hline HList & List of Hosts \\
\hline getUtilizationOfCPUMIPS & $\begin{array}{l}\text { Gets the utilization of } \\
\text { Hosts CPU in MIPS }\end{array}$ \\
\hline isHostOverUtilizedAfterAllocation & $\begin{array}{l}\text { Checks whether host is } \\
\text { overloaded after vm } \\
\text { allocaton. }\end{array}$ \\
\hline
\end{tabular}




\section{Algorithm 1: VM Mean and FFD Algorithm}

Input: HList, vmsToMigrate

Output: A virtual Machine Placement

1 Sort the vmsToMigrate in decreasing order of MIPS;

2 foreach VM in vmsToMigrate do

3 | totalVMUtilization = totalUtilization + VMUtilization 4calculate meanVMList

5 foreach VM in vmsToMigrate do

6 allocatedHost $\leftarrow$ null;

7 if NMUtilization lesser meanVMList then

8 \$ort the HList in decreasing order of power

9 foreach host in HList

10 if host is suitable for vm then

11 if getUtilizationOfCPUMIPS(host) \&\&

isHostOverUtilizedAffterAllocation(host, vm)

12 continue;

13 return host

14allocatedHost $\leftarrow$ host

15 return migrationMap

\section{TESTING ENVIRONMENT}

\section{A. Simulation setup}

The performance of VMs Mean and FFD algorithm compared against the three other VM Placement algorithms using CloudSim 3.0.3 toolkit. Simulation uses 800 heterogeneous host machine, half of which are HP ProLiant G4 and remaining half are HP ProLiant G5 servers. Energy consumption for G4 and G5 servers are listed in Table 2. Server's configuration are listed in Table 3. VM's configuration are listed in Table 4. For this simulation the data set we have used real world work load data provided from CoMon Project, a monitoring infrastructure for PlanetLab is listed in Table 5.

\section{B. Metrics for Measuring Performance}

To study the performance of the algorithm the following metrics are used.

1. Energy consumption - sum of the host machines energy consumption in the datacenter.

2. SLAV - are the violation in the Service Level Agreement's. If the requirements of the cloud consumers are not met, then SLA violation we get.

3. SLATAH - summing up all the violation for each host machine when the allocated MIPS is less than the requested MIPS.

4. PDM - average performance degradation caused in each host due to migrations.

5. VM migration numbers - VM's selected for migration from overloaded or under-loaded hosts.

6. ESV - combination of energy consumption and SLA violation.
Table 2Energy consumption by the selected servers at different load levels in Watts

\begin{tabular}{|l|l|l|l|l|l|l|}
\hline \multirow{2}{*}{$\begin{array}{l}\text { Machine } \\
\text { Type }\end{array}$} & \multicolumn{6}{|c|}{ Power Consumption Based on CPU } \\
& $0 \%$ & $20 \%$ & $40 \%$ & $60 \%$ & $80 \%$ & $100 \%$ \\
\hline $\begin{array}{l}\text { HP G4 } \\
\text { (Watt) }\end{array}$ & 86 & 92.6 & 99 & 106 & 112 & 117 \\
\hline $\begin{array}{l}\text { HP G5 } \\
\text { (Watt) }\end{array}$ & 93.7 & 101 & 110 & 121 & 129 & 135 \\
\hline
\end{tabular}

Table 3 Server'sConfiguration

\begin{tabular}{|l|l|}
\hline $\begin{array}{l}\text { Machine } \\
\text { Type }\end{array}$ & Description \\
\hline HP G4 & $\begin{array}{l}\text { 1860MIPS, 4 GB Ram, 1 GB Storage, 1 } \\
\text { GBnetwork bandwidth. }\end{array}$ \\
\hline HP G5 & $\begin{array}{l}\text { 2660 MIPS, 4 GB Ram, 1 GBStorage, 1 GB } \\
\text { network bandwidth. }\end{array}$ \\
\hline
\end{tabular}

\section{Table 4 VM's Configuration}

\begin{tabular}{|l|l|}
\hline VM types & Description \\
\hline $\begin{array}{l}\text { High-CPU } \\
\text { Medium } \\
\text { Instance }\end{array}$ & $\begin{array}{l}\text { 2500 MIPS, 870MB Ram, 2.5 GB Storage, } \\
100 \mathrm{Mbit} / \mathrm{s} \text { network bandwidth. }\end{array}$ \\
\hline $\begin{array}{l}\text { Extra Large } \\
\text { Instance }\end{array}$ & $\begin{array}{l}2000 \mathrm{MIPS}, 1740 \mathrm{MB} \text { Ram, 2.5 GB } \\
\text { Storage,100 Mbit/s network bandwidth. }\end{array}$ \\
\hline $\begin{array}{l}\text { Small } \\
\text { Instance }\end{array}$ & $\begin{array}{l}1000 \mathrm{MIPS}, 1740 \mathrm{MB} \text { Ram, 2.5 GB } \\
\text { Storage,100 Mbit/s network bandwidth. }\end{array}$ \\
\hline $\begin{array}{l}\text { Micro } \\
\text { Instance }\end{array}$ & $\begin{array}{l}500 \text { MIPS, 613 MB Ram, 2.5 GB } \\
\text { Storage,100 Mbit/s network bandwidth. }\end{array}$ \\
\hline
\end{tabular}

Table 5. Selected trace-based workloads.

\begin{tabular}{|l|l|l|l|}
\hline Workload & $\begin{array}{l}\text { No. of } \\
\text { VMs }\end{array}$ & Workload & $\begin{array}{l}\text { No. of } \\
\text { VMs }\end{array}$ \\
\hline 20110303 & 1052 & 20110403 & 1463 \\
\hline 20110306 & 898 & 20110409 & 1358 \\
\hline 20110309 & 1061 & 20110411 & 1233 \\
\hline 20110322 & 1516 & 20110412 & 1054 \\
\hline 20110325 & 1078 & 20110420 & 1033 \\
\hline
\end{tabular}

\section{Simulation result and Analysis}

CloudSim toolkit is used for the simulation. The proposed VMs Mean and FFD algorithm is compared with other three VM placement algorithms as described in Table 6. The proposed algorithm uses Local Regression (LR) as overload detection algorithm and Minimum Utilization (MU) as VM Selection policy. The simulation results shows that our proposed algorithm does maximizing the CPU Utilization with efficient energy consumption.

Discussion on Analysis of proposed algorithm along with other three VM placement algorithm are given below.

1. Energy consumption - the proposed algorithm consumes lesserenergy compared to remaining VM placement algorithm Figure 1 shows the result of the energy consumption.

2. SLA violation -the proposed algorithm shows low SLA violation ensures desired QoS. Figure 2 shows the result of the SLA violation. 
3. SLATAH - The AbsoluteCapacity algorithm have minimum SLA violation time per active host. Figure 3 shows the result of SLATAH.

4. PDM - the proposed algorithm shows less performance degradation due to migration. Figure 4 shows the result of PDM.

5. VM migration number - the proposed algorithm have less number of migrating VM's. Figure 5 shows the result of VM migration number.

6. ESV - the proposed have less combined energy and SLA violation. Figure 6 show the result.

From the above analysis the proposed algorithm performs better than the other three VM placement algorithm with respect to all the metrics used for measuring the performance. It proves that the proposed algorithm increase the host machine utilization by efficiently placing the VM's into few active Host's and also efficient energy consumption.

Table 6. Summary of all the VM placement Algorithms

\begin{tabular}{|l|l|l|l|l|}
\hline & MBFD & $\begin{array}{l}\text { Percent } \\
\text { ageUtil }\end{array}$ & $\begin{array}{l}\text { AbsoluteCa } \\
\text { pacity }\end{array}$ & $\begin{array}{l}\text { VM } \\
\text { Mean } \\
\text { and } \\
\text { FFD }\end{array}$ \\
\hline $\begin{array}{l}\text { Energy } \\
\text { Consumption } \\
(\mathrm{kWh})\end{array}$ & 176.18 & 115.88 & 114.10 & 110.69 \\
\hline $\begin{array}{l}\text { VM } \\
\text { Migration }\end{array}$ & 29553 & 16174 & 16775 & 12915 \\
\hline SLATAH & $8.31 \%$ & $7.18 \%$ & $5.71 \%$ & $9.41 \%$ \\
\hline PDM & $0.07 \%$ & $0.03 \%$ & $0.03 \%$ & $0.02 \%$ \\
\hline $\begin{array}{l}\text { SLAV } \\
\left(10^{-2}\right)\end{array}$ & 0.00595 & 0.00204 & $0.00167 \%$ & 0.00141 \\
\hline $\begin{array}{l}\text { ESV } \\
\left(10^{-2}\right)\end{array}$ & 1.04 & 0.23 & 0.19 & 0.15 \\
\hline $\begin{array}{l}\text { Average } \\
\text { SLA } \\
\text { Violation }\end{array}$ & $9.78 \%$ & $10.72 \%$ & $10.82 \%$ & $10.32 \%$ \\
\hline $\begin{array}{l}\text { Number of } \\
\text { Host } \\
\text { Shutdowns }\end{array}$ & 5573 & 840 & 938 & 814 \\
\hline
\end{tabular}

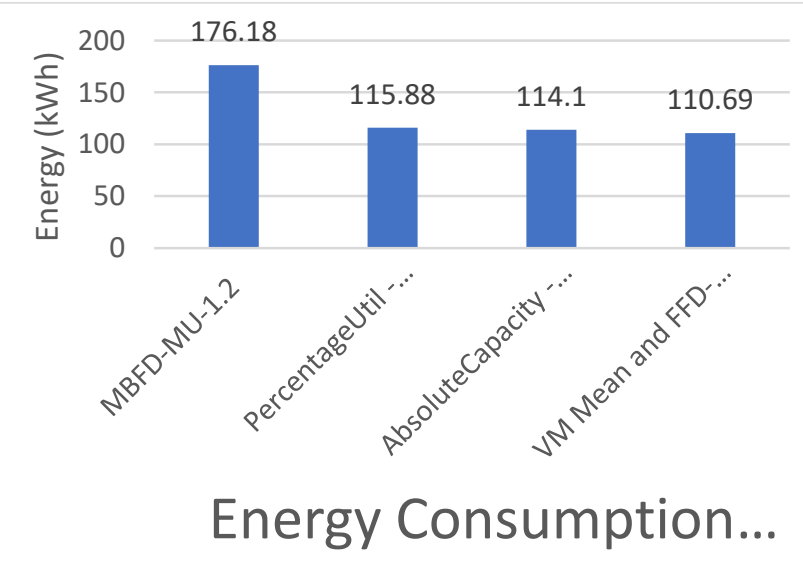

Figure 1 Energy Consumption Chart

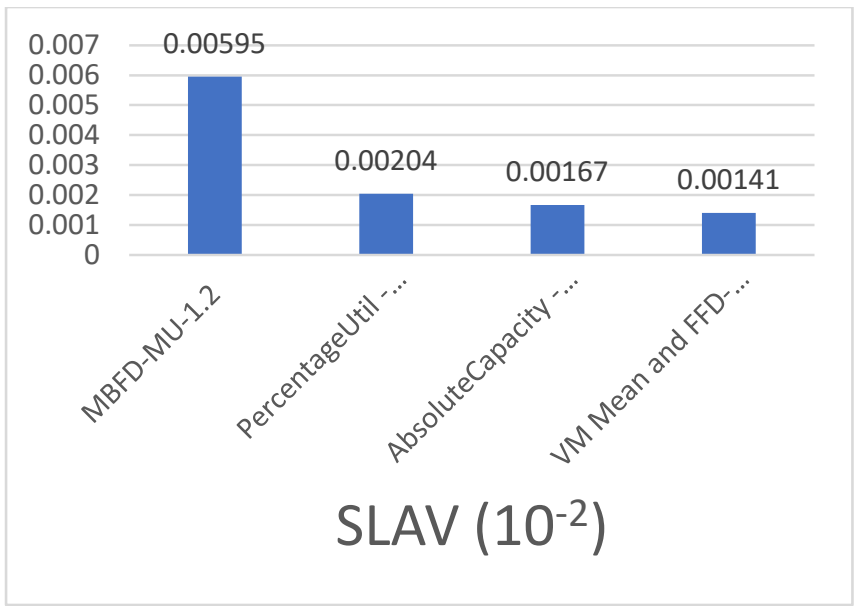

Figure 2 SLA Violation Chart

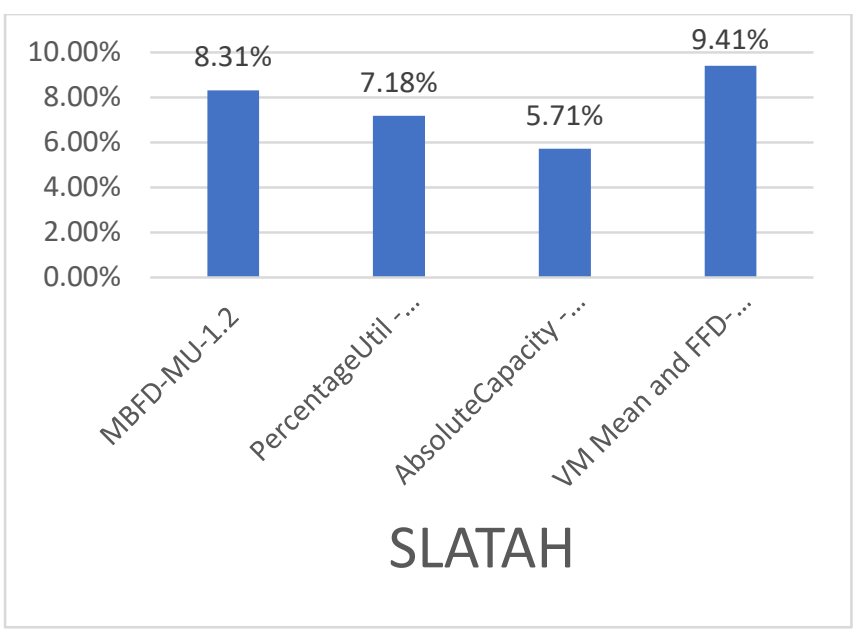

Figure 3 SLATAH Chart

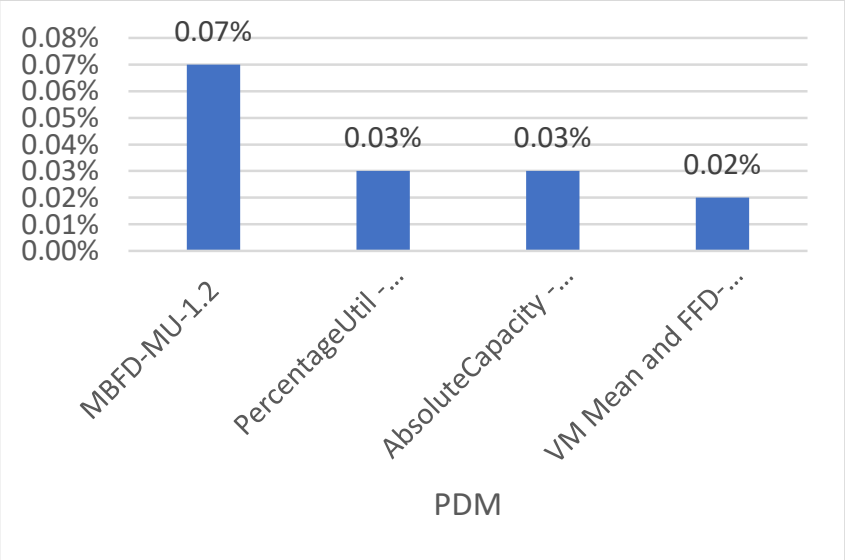

Figure 4 PDM Chart 


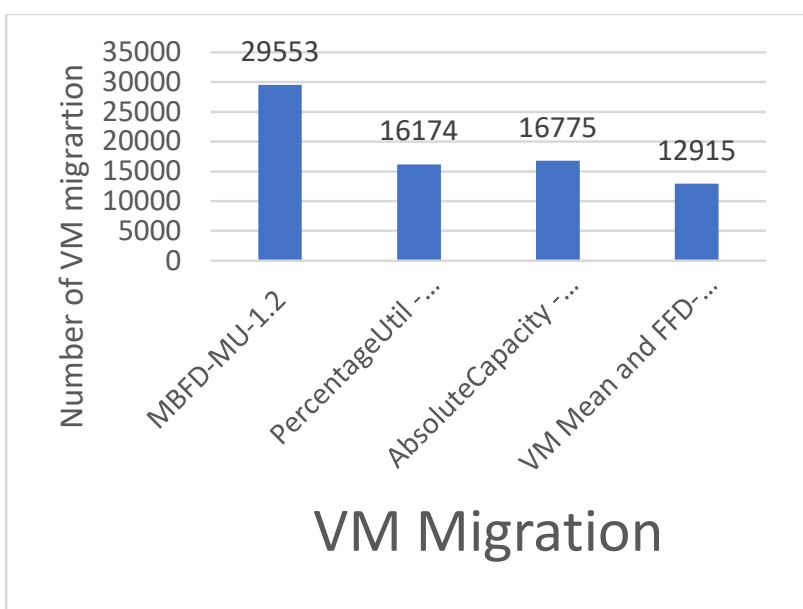

Figure 5 VM Migration Chart

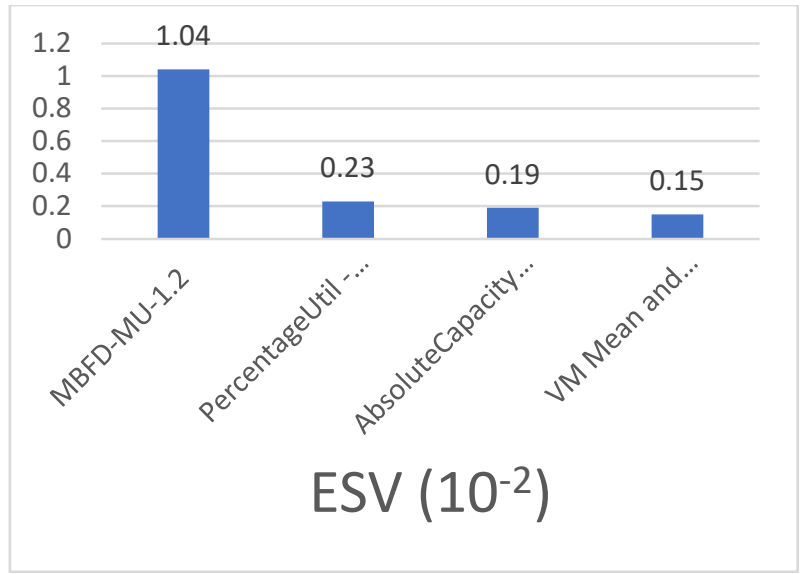

Figure 6 ESV Chart

\section{CONCLUSION}

The proposed VMs Mean and FFD algorithm calculates the mean value for the VM's selected for the migration. By using this mean value the lowest CPU utilization VM is selected and forwarded to the FFD algorithm. Then the FFD algorithm assigns the host machine for the VM's with the host machine having lowest power consumption. The proposed algorithm gives efficient VM placement, energy consumption and CPU utilization in cloud datacenter. Using this proposed algorithm we are able to achieve our main objective of maximize CPU Utilization and efficient energy consumption.

\section{REFERENCES}

1. Garima Batra, Harshita Singh, Ishu Gupta, Ashutosh Kumar Singh,"Best Fit Sharing and Power Aware (BFSPA) Algorithm for VMPlacement in Cloud Environment", 978-15090-6403-8/17/\$31.00 @2017 IEEE.

2. Zhou Zhou, Zhigang $\mathrm{Hu}$, and Keqin $\mathrm{Li}$, "Virtual MachinePlacement Algorithm for Both Energy-Awareness and SLAViolation Reduction in Cloud Data Centers", Hindawi PublishingCorporation Scientific Programming Volume 2016 Article ID5612039, $11 \quad$ pages http://dx.doi.org/10.1155/2016/5612039

3. Jing V. Wang, Nuwan Ganganath, Chi-Tsun Cheng, and Chi K.Tse, "A Heuristics-based VM Allocation Mechanism for CloudData Centers", 2017 IEEE International Symposium on Circuitsand Systems (ISCAS), Baltimore, MD, USA, 2017, pp. 1-4 isavailable at http://dx.doi.org/10.1109/ISCAS.2017.8050470

4. Aneeba Khalil Soomro, Mohammad Arshad Shaikh, Hameedullah Kazi, " FFD Variants for Virtual Machine Placement in Cloud Computing Data Centers", (IJACSA) International Journal of Advanced Computer Science and Applications, Vol. 8, No. 10 2017.
5. Pradeep Kumar, Dilbag Singh, Ankur Kaushik, "Power and Data Aware Best Fit Algorithm for Energy Saving in Cloud Computing", (IJCSIT) International Journal of Computer Science and Information Technologies, Vol. 5 (5) , 2014, 6712-6715.

6. Anton Beloglazov a,*, Jemal Abawajyb, Rajkumar Buyyaa, "Energy-aware resource allocation heuristics for efficient management of data centers for Cloud computing", 0167-739X/\$ - see front matter (C) 2011 Elsevier B.V. All rights reserved. doi:10.1016/j.future. 2011.04.017

7. L. Shi, J. Furlong, and R. Wang. "Empirical evaluation of vector bin packing algorithms for energy e cient data centers", In IEEE Symposium on Computers and Communications, pages $9\{15$, 2013.

8. Jay H. Sheth, Prof. Krunal N. Vaghela, "Technical Review on Live Virtual Machine Migration Techniques for Eucalyptus Cloud", Jay H. Sheth Int. Journal of Engineering Research and Applications, ISSN : 2248-9622, Vol. 5, Issue 3, ( Part -2) March 2015, pp.50-52.

9. Server Virtualization: A step toward Cost Efficiency and Business Agility, Avanade perspective, 2009.

10. Dabiah Ahmed Alboaneen1, Bernardi Pranggono and Huaglory Tianfield," Energy-aware Virtual Machine Consolidation for Cloud Data Centers2014 IEEE/ACM 7th International Conference on Utility and Cloud Computing.

11. Nicolo Maria Calcavecchia, Ofer Biran, Erez Hadad, and Yosef Moatti. VM placement strategies for cloud scenarios. In IEEE 5th International Conference on Cloud Computing (CLOUD), pages $852\{859,2012$

\section{AUTHORS PROFILE}

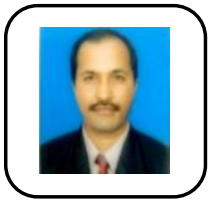

Sreenivasa B.L, Pursued his master degree Master of Science in Computer Science from Kuvempu University. Currently he is a research scholar in Bharathiar University Coimbatore and also as an Asst Professor in AIMIT, St Aloysius college, Mangalore. His interest of research is Cloud Computing.

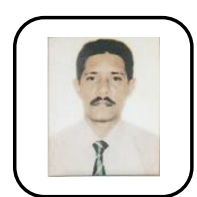

Dr S Sathyanarayana, Completed his BTech, Msc and Phd. His area of interest is Decision Support system and Cloud Computing, Relational Database Management Ssystems. 